\title{
cDNA microarray and bioinformatic analysis for the identification of key genes in Alzheimer's disease
}

\author{
$\mathrm{CHAO} \mathrm{GU}^{1 *}$ and TING SHEN ${ }^{2 *}$ \\ ${ }^{1}$ Department of Neurology, Longhua Hospital, Shanghai University of Traditional Chinese Medicine, Shanghai 200032; \\ ${ }^{2}$ Department of Emergency, Shanghai Municipal Hospital of Traditional Chinese Medicine, Shanghai 200071, P.R. China
}

Received August 7, 2013; Accepted November 5, 2013

DOI: 10.3892/ijmm.2013.1575

\begin{abstract}
In this study, gene expression profiles in peripheral blood monocytes from patients with Alzheimer's disease (AD) or mild cognitive impairment (MCI) were compared with those of healthy individuals to identify key differentially expressed genes (DEGs), in an effort to broaden our understanding of the pathogenesis of these diseases and identify potential therapeutic targets. The microarray data set GSE18309 was downloaded from Gene Expression Omnibus, including 3 AD, $3 \mathrm{MCI}$ and 3 normal control (NC) samples. Raw data were processed and differential analysis was performed using the $R$ multtest package. Two groups of comparisons (AD vs. NC and MCI vs. NC) were conducted and two groups of DEGs were acquired. The common DEGs were selected, for which functional enrichment analysis, as well as pathway enrichment analysis were performed to determine their roles in the development of the diseases in question. A total of 405 DEGs were identified in the AD vs. NC samples and 395 in the MCI vs. NC samples. A total of 60 common DEGs were obtained. Functional enrichment analysis revealed that the most common functions of the DEGs identified were response to nutrients, muscle contraction and cellular homeostasis. As shown by pathway enrichment analysis, the most common pathway associated with the DEGs identifed was the neuroactive ligandreceptor interaction pathway. A range of DEGs was identified in the present study, which may help to disclose the molecular mechanisms responsible for AD and may thus provide potential novel therapeutic strategies for Ad.
\end{abstract}

Correspondence to: Dr Chao Gu, Department of Neurology, Longhua Hospital, Shanghai University of Traditional Chinese Medicine, 725 South Wanping Road, Shanghai 200032, P.R. China E-mail: shdoctorchao@163.com

\section{${ }^{*}$ Contributed equally}

Key words: Alzheimer's disease, interaction network, mild cognitive impairment, microarray, functional enrichment analysis, pathway enrichment analysis

\section{Introduction}

Alzheimer's disease (AD) is a type of neurodegenerative disorder. It is characterized by memory loss, cognitive decline and persistent movement disorders, and is also accompanied by a series of psychotic symptoms (1). The increasing number of patients with the disease places a heavy burden on family and society. Therefore, there is an urgent demand for the early diagnosis and prevention of AD.

The pathogenesis of $\mathrm{AD}$ is very complex. A variety of factors have been found to be involved in $\mathrm{AD}$, such as cholinergic nerve abnormalities, metabolic disorders, free radicals and apoptosis, excitatory amino acid toxicity and genetic background (2). Despite their multifactorial etiopathogenesis, genetics plays a primary role in the progression of disease. Previous studies have identified four genes that may be linked to autosomal dominant or familial early onset AD. These are amyloid precursor protein (APP) (3), presenilin 1 (PS1) (4), presenilin 2 (PS2) (5) and apolipoprotein E (ApoE) (6). Some of the therapeutic approaches that have progressed to the clinical arena are the use of acetylcholinesterase inhibitors, nerve growth factors, non-steroidal anti-inflammatory drugs, estrogen and compounds, such as antioxidants, neuronal calcium channel blockers or anti-apoptotic agents (7). Moreover, the development of immunotherapic strategies may prove to be a therapeutic approach with promising results for the treatment of $\mathrm{AD}$.

Therefore, fundamental studies are required to provide novel and effective biomarkers for AD. Among the various technologies, microarray is a powerful and convenient tool which may aid in the search for biomarkers. Pasinetti (8) adopted cDNA microarray to search for molecular markers involved in the onset of AD dementia. Blalock et al (9) discovered major transcriptional and tumor suppressor responses in incipient AD by microarray correlation analyses. Maes et al (10) carried out transcriptional profiling of blood mononuclear cells from patients with $\mathrm{AD}$ by microarray analysis.

In present study, the cDNA microarray data of peripheral blood mononuclear cells from patients with $\mathrm{AD}$ were compared with those of normal control (NC) samples to discover the key genes associated with $\mathrm{AD}$. The data from transcriptome analysis of samples from patients with mild cognitive impairment (MCI) were also compared with those of NC samples, in order to identify early biomarkers for AD. 


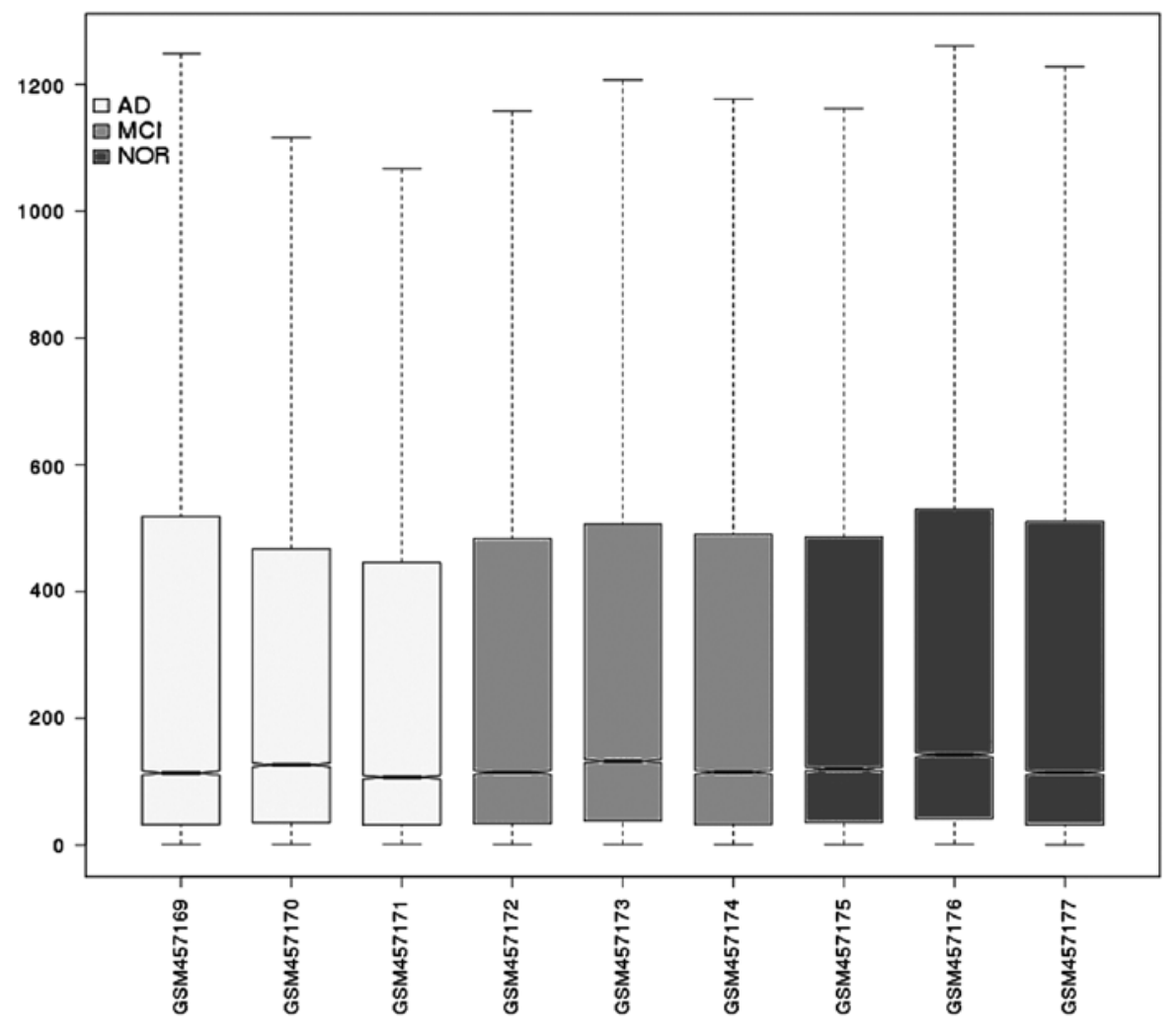

Figure 1. Box plot for normalized gene expression data. Alzheimer's disease (AD) samples are shown in white, mild cognitive impairment (MCI) samples in gray and normal controls in black. The medians (black lines) are almost at the same level, indicating a good performance standards.

\section{Materials and methods}

Microarray data. The microarray data set GSE18309 was downloaded from Gene Expression Omnibus, including $3 \mathrm{MCI}, 3 \mathrm{AD}$ and $3 \mathrm{NC}$ samples. The following platform was adopted: GPL570 [HG-U133_Plus_2] Affymetrix Human Genome U133 Plus 2.0 array to collect raw data.

Conversion of raw data. Raw data were converted into a recognizable format. After the missing values were imputed as previously described (11), the data were normalized, as described in a previous study (12). Two groups of comparisons were carried out: AD vs. NC and MCI vs. NC.

Differential analysis. Differential analysis (AD vs. NC and MCI vs. NC) was conducted with a t-test using the $R$ multtest package, as previously described (13). $\mathrm{P}<0.05$ and $\mid \log \mathrm{FCl}>1$ were set as the cut-off values to screen for differentially expressed genes (DEGs).

Construction of interaction network. Interactions between the two groups of DEGs were predicted with the STRING dagtabase (14), and then were visualized with Cytoscape, as previously described (15).

Functional enrichment analysis. The common DEGs were selected and functional enrichment analysis was then performed for these genes using the FuncAssociate web application, as previously described (16). A hypergeometric distribution-based test was applied and $\mathrm{P}<0.05$ was determined as the threshold.
Pathway enrichment analysis. Pathway enrichment analysis was performed with Fisher's exact test and the Expression Analysis Systematic Explorer (EASE) application, as previously described (17), which provided statistically significant GO terms. $\mathrm{P}<0.05$ was set as the threshold.

\section{Results}

Differentially expressed genes. Data normalization was caried out successfully (Fig. 1). A total of 405 DEGs were identified in the AD vs. NC samples and 395 DEGs in the MCI vs. NC samples. A total of $60 \mathrm{DEGs}$ were found to be common in both groups.

Interaction network. Interaction networks for the two groups of DEGs (AD vs. NC; MCI vs. NC) were constructed using the STRING and Cytoscape (Fig. 2). From the network, we were able to easily observe the locations of genes and thus identify key players in the networks. Several genes associated with inflammatory response were identified: chemokine $(\mathrm{C}-\mathrm{X}-\mathrm{C}$ motif) ligand (CXCL)11, CXCL3 and platelet factor 4 (PF4).

Functional enrichment analysis results. Common DEGs for $\mathrm{AD}$ and MCI were selected and analyzed using the FuncAssociate web application. The three most common functions of the DEGs identified were response to nutrients, muscle contraction and cellular homeostasis (Table I).

'Response to nutrients' (GO:0007584) was the most significant term (funcion) and four genes were found to be associated with this term: cholecystokinin A receptor (CCKAR), 
Table I. Three significant functional terms revealed by functional enrichment analysis.

\begin{tabular}{llll}
\hline Term ID & \multicolumn{1}{c}{ Function } & P-value & \multicolumn{1}{c}{ Genes } \\
\hline GO:0007584 & Response to nutrients & 0.006518 & CCKAR, IL6ST, CDKN2D, MAP1B \\
GO:0006936 & Muscle contraction & 0.008318 & P2RX6, SLC6A8, KCNQ1, GJC1 \\
GO:0019725 & Cellular homeostasis & 0.008333 & CCKAR, IL6ST, EGLN2, POU3F2, KCNQ1, KCNMB2
\end{tabular}

CCKAR, cholecystokinin A receptor; IL6ST, interleukin 6 signal transducer; CDKN2D, cyclin-dependent kinase inhibitor 2D; MAP1B, microtubule-associated protein 1B; P2RX6, purinergic receptor P2X, ligand-gated ion channel, 6; SLC6A8, solute carrier family 6 (neurotransmitter transporter, creatine), member 8; KCNQ1, potassium voltage-gated channel, KQT-like subfamily, member 1; GJC1, gap junction protein, gamma 1, 45 kDa; EGLN2, egl nine homolog 2 (C. elegans); POU3F2, POU class 3 homeobox 2; KCNMB2, potassium large conductance calcium-activated channel, subfamily $\mathrm{M}$, beta member 2 .

A

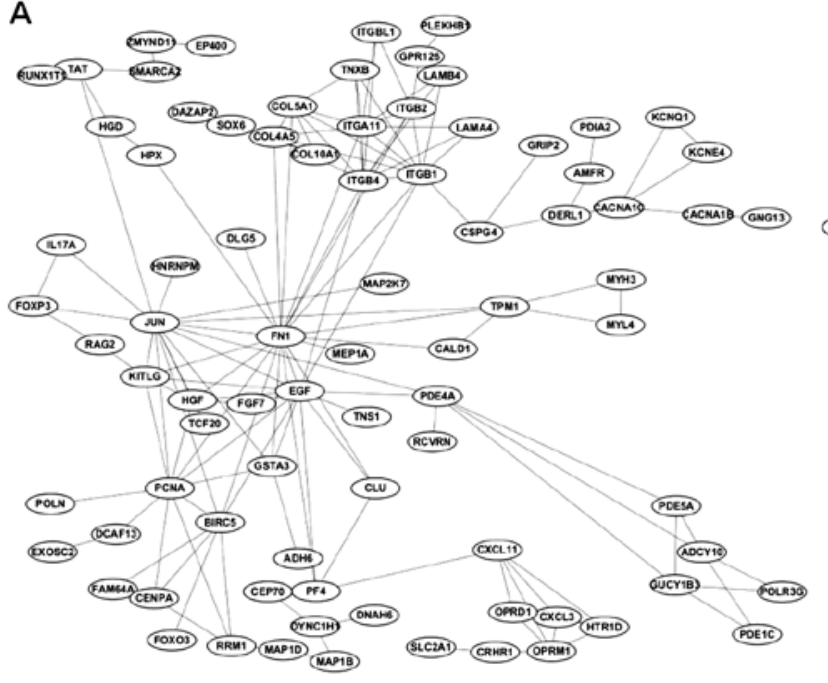

B

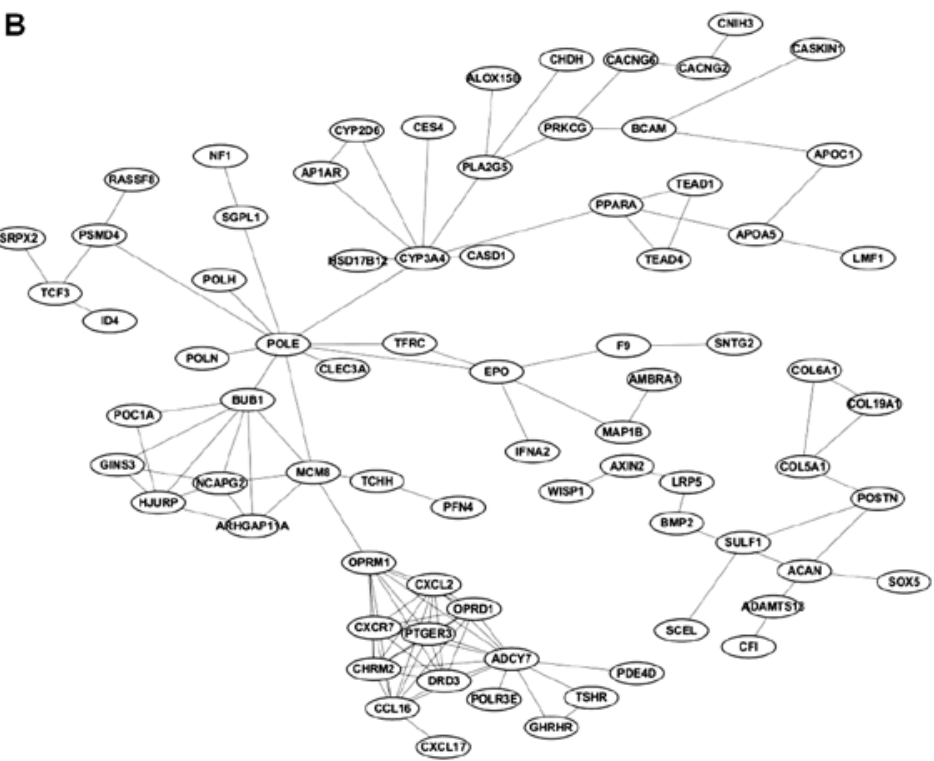

Figure 2. Interaction networks for differentially expressed genes (DEGs) from (A) Alzheimer's disease (AD) vs. normal controls (NC) and (B) mild cognitive impairment (MCI) vs. NC samples.

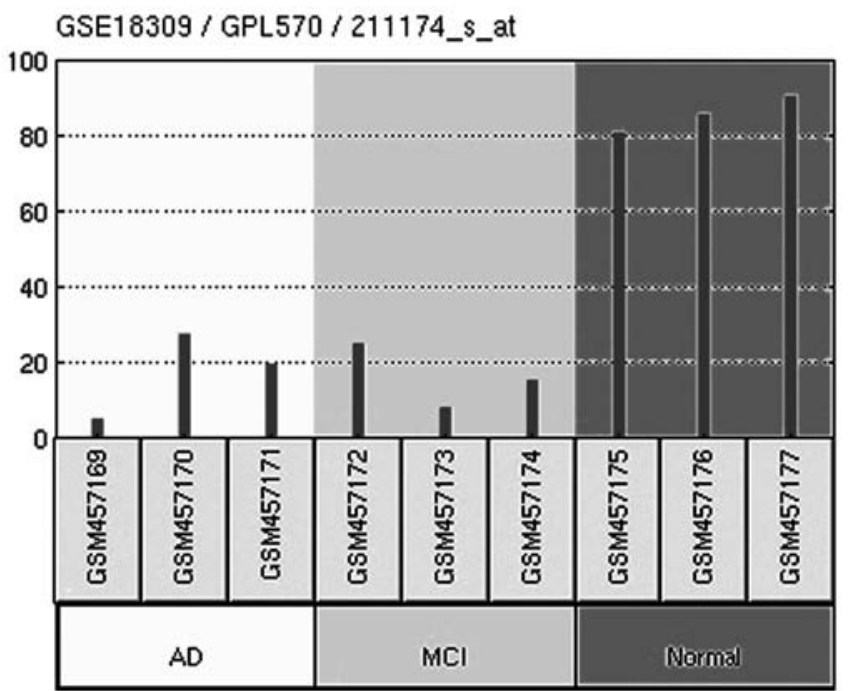

- expression value

Figure 3. Expression level of cholecystokinin A receptor (CCKAR) in each sample. Alzheimer's disease (AD) samples are shown in white, mild cognitive impairment (MCI) in gray and normal controls (NC) in black. interleukin 6 signal transducer (IL6ST), cyclin-dependent kinase inhibitor 2D (CDKN2D) and microtubule-associated protein 1B (MAP1B). Previous studies have demonstrated that cognitive impairment is related with a lack of nutrients. The dietary intake of certain nutrients or antioxidant supplements can prevent cognitive decline and the effects of dementia (18).

Pathway enrichment analysis results. The 60 common DEGs between the AD and MCI samples compared with NC samples were analyzed using the EASE application to identify the most common pathways associated with the DEGs identified. Teh most common pathway associated with the DEGs was the neuroactive ligand-receptor interaction pathway (hsa04080), associated with the opioid receptor, mu 1 (OPRM1), CCKAR, purinergic receptor $\mathrm{P} 2 \mathrm{X}$, ligand-gated ion channel, 6 (P2RX6) and opioid receptor, delta 1 (OPRD1) genes.

The CCKAR gene was found to be associated the most common functional term, as well the most common pathway, suggesting its important role in the development of AD. The downregulation of this gene was observed in the AD and MCI samples (Fig. 3). 


\section{Discussion}

Early diagnosis and prevention, as well as the development of molecular targeted therapy are of great importance to the treatment of AD. However, they require the identification of effective biomarkers. For this purpose, gene expression profiles in samples from patients with AD and MCI were compared with those of NC (healthy individuals). A total of 405 and 395 DEGs were revealed in the $\mathrm{AD}$ and $\mathrm{MCI}$ samples, respectively. Two interaction networks were then constructed to identify the key genes involved.

The association between inflammation and AD is very complicated (19). In the present study, several genes related to inflammation were identified. CXCL11 is a CXC member of the chemokine superfamily. It can induce a chemotactic response in activated T-cells and is the dominant ligand for CXC receptor-3. However, controversial roles have been found for the chemokine receptor CXCR3 and its ligands, CXCL9, CXCL10 and CXCL11, in the pathogenesis of neuroinflammatory diseases according to various studies (20). Several interactors of CXCL11 have been found in the network, such as CXCL3 and PF4. Endoplasmic reticulum (ER) stress is a novel neuronal trigger for inflammation and AD (21). The upregulation of CXCL3 has been observed in pateints with $\mathrm{AD}$ compared with healthy individuals, which can be explained by ER stress $(22,23)$. Platelets also play an important role in inflammatory processes and are thus involved in the development of AD (24). PF4 is one of the inflammatory signaling molecules secreted by platelets. Clusterin (CLU) interacts with PF4 and is a secreted chaperone. Protein misfolding is also closely linked with AD (25). In a genome-wide association study, Harold et al (26) found that variants at CLU and phosphatidylinositol binding clathrin assembly protein (PICALM) are associated with the development of AD. Similar findings were reported by Lambert et al (27). Braskie et al (28) further pointed out that variants within the CLU gene affect white matter microstructure in young adults.

In our study, 60 common DEGs were found in $\mathrm{AD}$ and $\mathrm{MCI}$. Three functional terms were significantly over-represented: response to nutrients, muscle contraction and cellular homeostasis. There may be a correlation between diet and the development of AD. Certain studies have suggested that a high intake of vitamins C, E (29), B6 and B12, as well as folate, unsaturated fatty acids and fish are related to a low risk of developing $\mathrm{AD}$; however, the results obtained are inconsistent $(30,31)$. Liu and Ames (32) reported that reducing mitochondrial decay with mitochondrial nutrients can delay and treat AD. Lefebvre et al (33) find that dysregulation of the nutrient/stress sensor, GlcNAcylation, is involved in the development of AD. MAP1B is thought to be involved in microtubule assembly, which is an essential step in neurogenesis (34). Aberrantly phosphorylated MAP1B has been detected in sites of neurofibrillary degeneration in the brains of patients with AD $(35,36)$. IL6ST (CD130) is a signal transducer shared by many cytokines, such as interleukin 6 (IL6) (37). Previous studies have indicated that abnormal IL6 activity contributes to the development of AD (38). Therefore, it may be a potential target to modulate signal transduction. In our study, pathway enrichment analysis revealed that the most common pathway associated with the DEGs identified was the neuroactive ligand-receptor interaction pathway.
The CCKAR was found to be associated with this pathway. It encodes a G-protein coupled receptor that binds non-sulfated members of the cholecystokinin (CCK) family of peptide hormones. It appears to be a major physiological mediator. In the central and peripheral nervous system, this receptor regulates satiety and the release of $\beta$-endorphin and dopamine (39). Wang et al (40) pointed out that there was an association between CCKAR polymorphism and visual hallucinogenesis in patients with Parkinson's disease. P2RX6 (P2X6) belongs to the family of $\mathrm{P} 2 \mathrm{X}$ receptors, which are ATP-gated ion channels and mediate rapid and selective permeability to cations. Köles et al (41) indicated that $\mathrm{P} 2 \mathrm{X}$ receptors are possible targets for therapeutic manipulations in disorders of the central nervous system. A previous study demonstrated blocking the purinergic P2X7 receptor exerts neuroprotective effects in an animal model of $\mathrm{AD}$ (42). Therefore, it is possible that P2X6 plays a role in the development of $\mathrm{AD}$. P2X receptor expression, mainly of that of P2X4 and P2X6 subtypes, has been detected in adult brains and during neuronal development. Da Silva et al (43) reported the alternative splicing of P2X6 receptors in the developing mouse brain and during in vitro neuronal differentiation.

In conclusion, a range of DEGs were identified in $\mathrm{AD}$ through this comparative study. These DEGs may play important roles in the development of AD. Further studies on these genes are required in order to fully elucidate their role in the pathogenesis of AD. Additionally, the common DEGs between $\mathrm{AD}$ and $\mathrm{MCI}$ are worthy of greater attention as they may be early biomarkers for $\mathrm{AD}$.

\section{Acknowledgements}

This study was supported by the Within Budget Project of Shanghai Municipal Education Commission (no. 2012JW34) and the Modernization of Traditional Chinese Medicine Project, Shanghai Municipal Science and Technology Commission (no. 12401904400).

\section{References}

1. Ferri CP, Prince M, Brayne C, et al: Global prevalence of dementia: a Delphi consensus study. Lancet 366: 2112-2117, 2005.

2. Ballard C, Chalmers K, Todd C, et al: Cholinesterase inhibitors reduce cortical $A \beta$ in dementia with Lewy bodies. Neurology 68 : 1726-1729, 2007.

3. O'Brien RJ and Wong PC: Amyloid precursor protein processing and Alzheimer's disease. Annu Rev Neurosci 34: 185-204, 2011.

4. Duff K, Eckman C, Zehr C, et al: Increased amyloid-beta42(43) in brains of mice expressing mutant presenilin 1. Nature 383 : 710-713, 1996.

5. Jayadev S, Case A, Eastman AJ, et al: Presenilin 2 is the predominant $\gamma$-secretase in microglia and modulates cytokine release. PloS One 5: e15743, 2010.

6. Strittmatter WJ, Saunders AM, Schmechel D, et al: Apolipoprotein E: high-avidity binding to beta-amyloid and increased frequency of type 4 allele in late-onset familial Alzheimer disease. Proc Natl Acad Sci USA 90: 1977-1981, 1993.

7. Parihar M and Hemnani T: Alzheimer's disease pathogenesis and therapeutic interventions. J Clin Neurosci 11: 456-467, 2004.

8. Pasinetti GM: Use of cDNA microarray in the search for molecular markers involved in the onset of Alzheimer's disease dementia. J Neurosci Res 65: 471-476, 2001.

9. Blalock EM, Geddes JW, Chen KC, Porter NM, Markesbery WR and Landfield PW: Incipient Alzheimer's disease: microarray correlation analyses reveal major transcriptional and tumor suppressor responses. Proc Natl Acad Sci USA 101: 2173-2178, 2004. 
10. Maes OC, Xu S, Yu B, Chertkow HM, Wang E and Schipper HM Transcriptional profiling of Alzheimer blood mononuclear cells by microarray. Neurobiol Aging 28: 1795-1809, 2007.

11. Troyanskaya O, Cantor M, Sherlock G, et al: Missing value estimation methods for DNA microarrays. Bioinformatics 17 520-525, 2001.

12. Fujita A, Sato JR, Rodrigues Lde O, Ferreira CE and Sogayar MC: Evaluating different methods of microarray data normalization. BMC Bioinformatics 7: 469, 2006.

13. Pollard KS, Dudoit S and van der Laan MJ: Multiple testing procedures: $\mathrm{R}$ multtest package and applications to genomics. In: Bioinformatics and Computational Biology Solutions Using $R$ and Bioconductor. Gentleman R, Carey V, Huber W, Irizarry R and Dudoit S (eds). Springer, New York, pp251-272, 2005.

14. Szklarczyk D, Franceschini A, Kuhn M, et al: The STRING database in 2011: functional interaction networks of proteins, globally integrated and scored. Nucleic Acid Res 39: D561-D568, 2011.

15. Shannon P, Markiel A, Ozier O, et al: Cytoscape: a software environment for integrated models of biomolecular interaction networks. Genome Res 13: 2498-2504, 2003

16. Berriz GF, Beaver JE, Cenik C, Tasan M and Roth FP: Next generation software for functional trend analysis. Bioinformatics 25 3043-3044, 2009.

17. Hosack DA, Dennis G Jr, Sherman BT, Lane HC and Lempicki RA: Identifying biological themes within lists of genes with EASE. Genome Biol 4: R70, 2003.

18. Balk EM, Raman G, Tatsioni A, Chung M, Lau J and Rosenberg IH: Vitamin B6, B12, and folic acid supplementation and cognitive function: a systematic review of randomized trials. Arch Intern Med 167: 21-30, 2007.

19. Wyss-Coray T: Inflammation in Alzheimer disease: driving force, bystander or beneficial response? Nat Med 12: 1005-1015, 2006.

20. Müller M, Carter S, Hofer MJ and Campbell IL: Review: The chemokine receptor CXCR3 and its ligands CXCL9, CXCL10 and CXCL11 in neuroimmunity - a tale of conflict and conundrum. Neuropathol Appl Neurobiol 36: 368-387, 2010.

21. Salminen A, Kauppinen A, Suuronen T, Kaarniranta K and Ojala J: ER stress in Alzheimer's disease: a novel neuronal trigger for inflammation and Alzheimer's pathology. J Neuroinflammation 6 : $41,2009$.

22. Gargalovic PS, Gharavi NM, Clark MJ, et al: The unfolded protein response is an important regulator of inflammatory genes in endothelial cells. Arterioscler Thromb Vasc Biol 26 2490-2496, 2006.

23. Zhang K and Kaufman RJ: From endoplasmic-reticulum stress to the inflammatory response. Nature 454: 455-462, 2008.

24. Catricala S, Torti M and Ricevuti G: Alzheimer disease and platelets: how's that relevant. Immun Ageing 9: 20, 2012.

25. Casserly I and Topol E: Convergence of atherosclerosis and Alzheimer's disease: inflammation, cholesterol, and misfolded proteins. Lancet 363: 1139-1146, 2004.

26. Harold D, Abraham R, Hollingworth P, et al: Genome-wide association study identifies variants at CLU and PICALM associated with Alzheimer's disease. Nat Genet 41: 1088-1093, 2009.

27. Lambert JC, Heath S, Even G, et al: Genome-wide association study identifies variants at CLU and CR1 associated with Alzheimer's disease. Nat Genet 41: 1094-1099, 2009.
28. Braskie MN, Jahanshad N, Stein JL, et al: Common Alzheimer's disease risk variant within the CLU gene affects white matter microstructure in young adults. J Neurosci 31: 6764-6770, 2011.

29. Morris MC, Evans DA, Bienias JL, et al: Dietary intake of antioxidant nutrients and the risk of incident Alzheimer disease in a biracial community study. JAMA 287: 3230-3237, 2002.

30. Luchsinger JA and Mayeux R: Dietary factors and Alzheimer's disease. Lancet Neurol 3: 579-587, 2004.

31. Luchsinger JA, Noble JM and Scarmeas N: Diet and Alzheimer's disease. Curr Neurol Neurosci Rep 7: 366-372, 2007.

32. Liu J and Ames BN: Reducing mitochondrial decay with mitochondrial nutrients to delay and treat cognitive dysfunction, Alzheimer's disease, and Parkinson's disease. Nutr Neurosci 8: 67-89, 2005.

33. Lefebvre T, Dehennaut V, Guinez C, et al: Dysregulation of the nutrient/stress sensor O-GlcNAcylation is involved in the etiology of cardiovascular disorders, type-2 diabetes and Alzheimer's disease. Biochim Biophys Acta 1800: 67-79, 2010.

34. Gordon-Weeks PR and Fischer I: MAP1B expression and microtubule stability in growing and regenerating axons. Microsc Res Tech 48: 63-74, 2000.

35. Ulloa L, Montejo de Garcini E, Gómez-Ramos P, Morán MA and Avila J: Microtubule-associated protein MAP1B showing a fetal phosphorylation pattern is present in sites of neurofibrillary degeneration in brains of Alzheimer's disease patients. Brain Res Mol Brain Res 26: 113-122, 1994.

36. Hasegawa M, Arai T and Ihara Y: Immunochemical evidence that fragments of phosphorylated MAP5 (MAP1B) are bound to neurofibrillary tangles in Alzheimer's disease. Neuron 4: 909-918, 1990.

37. García-Bueno B, Caso JR and Leza JC: Stress as a neuroinflammatory condition in brain: damaging and protective mechanisms. Neurosci Biobehav Rev 32: 1136-1151, 2008.

38. Hampel H, Teipel SJ, Padberg F, et al: Discriminant power of combined cerebrospinal fluid tau protein and of the soluble interleukin- 6 receptor complex in the diagnosis of Alzheimer's disease. Brain Res 823: 104-112, 1999.

39. Huppi K, Siwarski D, Pisegna J and Wank S: Chromosomal localization of the gastric and brain receptors for cholecystokinin (CCKAR and CCKBR) in human and mouse. Genomics 25 727-729, 1995

40. Wang J, Si YM, Liu ZL and Yu L: Cholecystokinin, cholecystokinin-A receptor and cholecystokinin-B receptor gene polymorphisms in Parkinson's disease. Pharmacogenetics 13: 365-369, 2003

41. Köles L, Furst S and Illes P: P2X and P2Y receptors as possible targets of therapeutic manipulations in CNS illnesses. Drug News Perspect 18: 85-101, 2005.

42. Ryu JK and McLarnon JG: Block of purinergic P2X7 receptor is neuroprotective in an animal model of Alzheimer's disease. Neuroreport 19: 1715-1719, 2008

43. da Silva RL, Resende RR and Ulrich H: Alternative splicing of P2X6 receptors in developing mouse brain and during in vitro neuronal differentiation. Exp Physiol 92: 139-145, 2007. 